Voix et Images

voixetimages

\title{
" Le Québec est en creux » Neige noire de Hubert Aquin
}

\section{Monique Roy-Gans}

Volume 7, numéro 3, printemps 1982

Anne Hébert

URI : https://id.erudit.org/iderudit/200348ar

DOI : https://doi.org/10.7202/200348ar

Aller au sommaire du numéro

\section{Éditeur(s)}

Les Presses de l'Université du Québec

\section{ISSN}

0318-9201 (imprimé)

1705-933X (numérique)

Découvrir la revue

\section{Citer cet article}

Roy-Gans, M. (1982). " Le Québec est en creux " Neige noire de Hubert Aquin.

Voix et Images, 7(3), 553-569. https://doi.org/10.7202/200348ar d'utilisation que vous pouvez consulter en ligne.

https://apropos.erudit.org/fr/usagers/politique-dutilisation/ 


\title{
"Le Québec est en creux» Neige noire de Hubert Aquin
}

\author{
par Monique Roy-Gans, University of California
}

* Jusqu'à présent dans le film, on peut dire que le Québec est en creux. Son éclipse récurrente fait penser à l'absence d'une présence, à un mystère inachevé. . In

Nerge noire. p. 136

Toute étude critique de Neige noire de Hubert Aquin se heurte d'emblée à un chassé-croisé de formes complexes et fuyantes. Cuvre intensément moderne, essentielle dans sa quête, ce roman-scénario est le mıroir cinétıque des multiples formes du réel et de l'imaginaire. Si bien que c'est unıquement à partir de ce délire formel, de ces adébris plus ou moins rattachés à la coulée d'où ils proviennent, ' que le lecteur va pouvoır reconstituer la dialectıque fondamentale de l'cuvre. Lire Neige noire est avant tout tenter de reconstruire cette dialectique, travail de reconstruction auquel nous invite l'auteur dans le commentaıre discursıf ou métalinguistique qui alterne avec l'aspect narratıf ou fictionnel du roman: le scénario en cours d'élaboration. C'est là une plangée vertigineuse jusqu'aux rites les plus norrs où s'exorcisent l'impurssance et l'inefficacité pour arriver au mythe qui fonde toute activité humaine. Dans Neige noire, c'est une conscience paralysée par la colonisation. celle de Nicolas Vanesse qui, en quête du devenir historique, s'achemine sur cette trajectorre douloureuse. Au cours de l'itinéraire, ce dernier découvre le paradoxe essentiel de I'homme, paradoxe tıré de Kierkegaard que l'on trouve en exergue à Neige noire: “ Je dois maintenant à la fois être et ne pas ètre." De sorte que tout homme désireux de s'affirmer comme ètre doit d'abord séjourner dans sa négativité pour ensuite la nier, travail de "négation de la négation," qui est l'essentiel du devenir hégélien.

Chez Nicolas, cependant, ce devenır se joue non pas sur le "theaatre illuminé " où se déroule la "parabole" de toutes les cuvres humaines, mais sur cette autre parabole, section d'un cône renversé, c'est-à-dire dans al'édifice fêlé" d'une conscience colonisée. C'est pourquoi le roman est présenté par 
l'auteur comme xune étude ininterrompue sur Undensacre (p. 196), symbole légendaire dont l'existence est douteuse et qui renferme le devenir québécois. Undensacre, c'est le lieu où, selon une tradition mythologique, serait enterré le frère jumeau de Hamlet, Fortinbras. C'est à partir de l'échec de Hamlet que Fortinbras, grâce d̀ sa détermination, va reconquérir son identité ainsi que ses droits sur son pays. Fortinbras se démasque à la mort de.Claudius et de Hamlet pour devenir Prince du Danemark; en d'autres mots, il nie son manque d'être. alors que Hamlet, héros tragique, résume son impuissance et son inefficacité dans te premier vers de son célèbre soliloque: * To be or not to be, that is the question." C'est dans cette hésitation fatale entre le non-être et l'être que Hamlet épouse son Destin tragique. Effectivement, l'unique choix de I'homme n'est pas entre «ètre» et * ne pas être» mais plutôt entre agir, qui est le travail de la conscience historique sur son essentielle négativité - - à la fois être et ne pas être» - et ne pas agir, qui est le refus d'intégrer le négatif à l'être. Dès que I'homme, à l'exemple de Fortinbras, opte pour l'action, il est en mesure de récupérer une identité qui, autrement, demeure dissimulée derrière le masque du "manque d'être” hégélien. Celui en revanche qui choisit de ne pas agir embrasse l'échec hamlétien et, de ce fait, se voue au Destin tragique. Fortinbras et Hamlet, deux figures shakespeariennes, l'une anti-tragique, l'autre tragique, comme des doubles qui se cherchent, s'affrontent en Nicolas dans une tentative de conciliation. C'est là une tâche d'intégration à l'histoire, tâche qui exige une transformation de l'angoisse de la négativité hamlétienne en devenir historique de Fortinbras, genèse toujours à refaire qui est le propre de l'homme et son unique victoire.

C'est dans cette perspective que j'ai choisi d'étudier Neige noire. La quête de Nicolas, en ce sens, se résume en une recherche du mouvement dialectique qui mène à la conciliation; démarche qui, selon Hegel, est "la saisie des opposés dans leur unité, ou du positif dans le négatif. ${ }^{2}$ Chez Nicolas, cela consiste à exorciser l'impuissance et l'inefficacité de Hamlet afin d'amener l'angoisse métaphysique de ce dernier à une détermination dont il fera sortir le contenu et le résultat positifs. C'est là un processus par lequel la détermination devient "développement et progression immanente." 3 Et c'est en Fortinbras, dont il joue le rôle dans la pièce de Shakespeare, que Nicolas va trouver l'efficacité indispensable à son entreprise. Cela est évident dès les premières pages du roman: "Les plans de Nicolas sont figés, tandis que son reflet dans le miroir est cinétique. Le seul synchronisme survient quand il dit: 'Où donc est ce spectacle?' ["Where is this sight?" (Ham/et, V, ii)]. Aussitôt dit, les deux images se décollent l'une de l'autre, et instaurent, de ce fait, une défocalisation psychologique...» (p. 15). Lorsque Nicolas décide de quitter le travail de comédien pour devenir scénariste-réalisateur, de passer pour ainsi dire de la répétition a la création, il manifeste une volonté d'abolir cette défocalisation paralysante de sa conscience; il choisit la voie du devenir historique qui mène au lieu du spectacle.

Or, opter pour le devenir humain, c'est surtout initier le processus dialectique qui est celui de l'objectivité ou de la négativité à l'égard de son 
Soi qui devient autre. Toutefois, cette auto-objectivation de la conscience n'est pas une extériorisation mais plutôt al'âme propre du contenu qui produit organiquement ses branches et ses fruits. " Dans la première partie de Neige noire, cet «être-autren se manifeste comme les doubles faces du tragique, c'est-à-dire, la négativité impuissante ou coupable de l'esprit hamlétien que figure Sylvie Vanesse, épouse de Nicolas, et celle d'Ophélie, double en miroir de Hamlet que représente Linda Noble. l'actrice qui en joue le rôle dans la pièce de Shakespeare. ${ }^{5}$ Linda se distingue cependant d'Ophélie en ce qu'elle est «déterminée»: "Personnage écran, elle reflète, mais rien ne passe à travers elle, surtout pas la lumière (p. 23). Linda, dans la perspective hégélienne, est * une figure concrète dans l'être-là total de laquelle une détermination est dominante. ${ }^{\circ}$ Mais elle est une détermination en attente du mouvement médiateur par lequel Linda sera en mesure d'échapper au Destin tragique d'Ophélie pour finalement s'intégrer à la conscience de Nicolas. Dans la première partie du roman. Linda Noble demeure donc "confinée à son masque". attendant que Sylvie, cet autre cètre-autre», soit supprimée afin de donner lieu a l'esprit efficace de Fortinbras qui sera figuré par la Norvégienne, Eva Vos. Double incomplet qui « joue sans àme, dira Nicolas (p. 169), Linda est /être-là de la conscience de Nicolas, le Dasein dans lequel "l'imprévisible peut arriver" (p. 23). Sylvie Vanesse et Linda Noble, toutes deux produits organıques d'une même conscience objectifiée, doubles de Nicolas, ne parviennent jamais à se synchroniser: "les deux images n'arrivent pas à être au foyer en mème temps» (p. 19).

C'est en Sylvie Vanesse que la conscience hamlétienne se manifeste de la façon la plus pure. Héritière en quelque sorte du «nom blessé » que Hamlet aurait laissé derrière lui [ $\mathrm{O}$ God, Horatio, what a wounded name, , Things standing thus unknown, shall live behind me!" (Hamlet, $V$, ii)]. Sylvie Vanesse conjugue l'identité double du passé. En elle se dissımule l'innocence du temps où elle était Sylvie Lewandowski, identité originelle qu'elle cherche vaınement dans les bras de son père, Michel Lewandowski. "ll y a de la neige partout dans mon enfance," dira-t-elle. "Cela n'arrètait pas. Moi. j'étais aveuglée par les gros flocons... Et quand je n'en pouvais plus, tu me prenais dans tes bras... Tu tè souviens, papa? (p. 219). Cependant, Sylvie Lewandowski est devenue Syıvie Dubuque, nom qu'elle adopte pour dissımuler sa liaison coupable avec son père mais qui n'est en fin de compte qu'une substitution du "pare $/$ au mème»car c'est sous ce nom (et celui de Vanesse) qu'elle souille l'innocence de jadis.

Le rapport que Sylvie entretient avec le passé n'est pas un rapport d'espérance mais bien le rapport infernal de ce que Kierkegaard appelle le "ressouvenir éternel de la conscience de la faute," selon lequel "l'exıstant s'accroche en quelque sorte à la félicité par le fil le plus ténu à l'aıde d'une possibilité qui sombre sans cesse...,n? c'est-á-dire, dans le cas de Sylvie. par l'inceste. Miroir cedipien de l'être. l'existence de Sylvie est transformée en une sorte d'“adhésion moite au réel»; elle n'est capable de se présenter à elle-mème ni dans le présent ni dans l'avenir, car elle a déjà vécu [ SSyivie 
n'était pas, elle a été (p. 131)], ni dans le passé qui, étant justement ce qu'elle espère ressaisir, n'a pas encore eu lieu. L'existant, dans de telles circonstances, est a toujours en enfantement, sans jamais enfanter... il n'a point de présent auquel se rattacher, point de passé après lequel soupirer, car son passé n'est pas encore venu, point d'avenir qu'il puisse espérer, car.son avenir est déjà révolu. ${ }^{8}$ Nayant pour ainsi dire pas de temps, Sylvie Vanesse, juste avant sa mort, demande à Nicolas : « Comment savoir quand ce sera demain ? (p. 109).

Enlisée dans le passé. génitrice d'impuissance fon se souvient qu'elle blesse Nicolas au pénis), image par trop évidente du narcissisme québécois tant déploré par l'auteur. Sylvie bloque le progrès et sera donc éliminée. Personnage essentiellement tragique - elle se distingue par ses « muscles du chagrin exceptionnels» (p. 135) - Sylvie mourra sous le couteau de Nicolas, comme Hamlet sous l'épée de Laerte. Comme Hamlet, et c'est l'auteur qui l'écrit dans Trou de mémoire, "le Québec, c'est cette poignée de comédiens bègues et amnésiques qui se regardent et sinterrogent du regard et qui semblent hantés par la platitude comme Harnilet par le spectre. ${ }^{9}$ Éliminer Sylvie, victime du Sollen hégélien, c'est bien, dans cette perspective, exorciser la tragédie de «ce pur devoir ètre qui exige la conciliation sans jamais y atteindre... ${ }^{10}$ C'est pourquoi, dans la première partie du roman, Sylvie a est la structure porteuse de l'cuure. Pour quelque temps encore, tout se greffe sur sa peau. tout se mesure par rapport à elle (p. 46). Or, mesurer tout par rapport à Sylvie, c'est en somme réduire tout ce qu'elle touche "plutôt à un moulage de plâtre qu'à une représentation mobile de ce qui se meut» (p. 45), c'est transformer les choses, y compris l'écriture, en un amagma informe" où ace qui est présenté... ne représente plus rien» (p. 18). Même phénomène d'entropie que l'on retrouve a Montréal, ville où "le mouvement de la vie est réduit à une stase languissante* (p. 7). Aussi le scénario va-t-il demeurer en état d'esquisse jusqu'a la mort de Sylvie: "Depuis que j'ai quitté Montréal," dira Nicolas a. Eva Vos, "je n'ai fait qu'ajouter des notes et des gloses à mon synopsis..." (p. 141).

Si le narrateur est incapable de capter Sylvie par l'écriture, c'est qu'elle est aussi "l'origine et le terme de toutes les successions, et le symbole allusif de la durée» (p. 46). Or, toute référence à la succession dans le cadre de la durée est une intervention de l'idée d'espace dans le flux temporel. Cette spatialisation ad nauseam du Temps que l'auteur confère à Montréal où "le temps n'est pas cette entité extensible ou plus ou moins compressible» (p. 185), est une conception quantitative du temps continu. Mesurable et prévisible. Mais, nous dira l'auteur, "ce qui est prévisible ne fait que se développer dans la sérénité de la raison. La continuité, c'est un enchaînement sans ellipses, sans bonds, sans hoquets, sans coupures, sans excursus latéraux. L'existence, aux yeux du souvenir, a parfois ce caractère anormalement continu, et les élémerits les plus discordants deviennent ainsi agencés, par un effet de perspectives, selon la linéarité la plus pure $\nsim(p .48$ ). C'est là le temps homogène dont parle Bergson, " cette "quatrième dimension de l'espace" selon laquelle. 
grâce au souvenir, la conscience organise, puis aligne les phases successives de la vie consciente. Ce qui signifie, en somme, que le temps homogène n'est pas une intégration des états antérieurs avec l'état actuel de la conscience. "pénétration mutuelle" qui est essentielle à une conception qualitative de la durée où "chaque élément est représentatif du tout. ne s'en distingue et ne s'en isole que pour une pensée capable d'abstraire."12 Dans la représentation quantitative de la durée, les états successifs de la conscience sont juxtaposés ou spatialisés de sorte qu'il est possible de les concevoir l'un à côté de l'autre. Simultanéité qui transforme le passé en une étendue dont les phases se succèdent l'une à l'autre sur une ligne continue qui n'est somme toute qu'une représentation symbolique d'une durée homogène. Alors que la durée hétérogène, dans sa pureté originelle. non spatialisée, "prévaut a jamais sur les modes de successions" (p. 40).

Tout autre est la fragmentation du flux temporel que l'on trouve dans le scénario. Pour ainsi dire atrophié temporairement par Sylvie, ce synopsis. où "rien n'est appelé à durer," où tout est chronométré par une série de cadrages, offre néanmoıns les “ débris nécessaires à une conception qualitatıve de la durée». En effet, c'est sur ce devenir qui n'est qu'une "séquence de séquence," qu'une "cendre imagée" (pp. 40-41), que Nicolas va recomposer "la contexture du temps originaire» (p. 48). Là oú Linda Noble, nous le disıons ci-dessus, est le lieu où "l'imprévisible peut arriver, " le lieu d'une détermination en attente, le scénario, bien qu'en état d'esquisse, représente le «discontinu... qui se réfère à des ruptures ou à des extensions imprévues" (p. 48). Car "aucune fiction ne peut masquer cet 'ordre' imprévisıble de l'existence. L'imprévisible, semble-t-il, contient la formule basale de toute representation factice de la vie» (p. 48). Et c'est uniquement à partır de cette "spécificité fluente" que peut s'exercer l'action du cinéaste.

En revanche, c'est selon un "schéma préconçu" que se structure Montréal, "Synonyme d'encombrement," c'est le lieu où toute activité demeure emprisonnée dans un cercle infernal. Tout au plus, peut-on s'échapper de cette "métrique urbaine» pour accomplir ailleurs l'action libératrıce. Ce thème revient dans tous les romans d'Hubert Aquin. De l'écrivain prisonnier à Montréal qui, dans Prochain épisode, se projette "entre les plages éclatées du Lac Léman... dans l'eau mème du Rhône agrandi" où le narrateur "plonge inlassablement à la recherche de son cadavre, "13 à Nicolas Vanesse qu immole Sylvie sur les sommets du Spitzbergen en Norvège dans Neige noire. c'est ce même travail de désaliénation quı sous-tend l'œeuvre aquinienne. C'est sans doute pourquoi le port de Montréal joue un rôle privilégié dans ce roman. Linda Noble, entrevue par Eva Vos à la télévision, rencontrera cette dernière pour la première fois dans une "pıèce à plafond surbaıssé quı donne sur le port de Montréal" (p. 230), pièce qui est le bureau de Nicolas. C'est aussi devant l'immeuble de l'administration du port de Montréal que Sylvie va révéler la véritable nature de l'impuissance qui la paralyse. En quète d'une échappatoire au mouvement entropique qui lemporte, elle ne choisit pas la voie que 
recherchent Linda et Eva et qui mènera à l'unité symbolique qu'elles atteindront a la fin de Neige noire, ni même le devenir qui attend Nicolas en Norvège, pays dont l'autonomie a été acquise au début du siècle. Sylvie Vanesse manifeste le désir de «rouler jusqu'à Natchez-under-the-Hill, cet autre Montréal en quelque sorte : Natchez, ville historique sur les rives du Mississippi, ancienne possession française (Fort Rosalie), conquise trois fois - par les Natchez, les Anglais, puis les Espagnols - finalement intégrée aux États-Unis. Centre puissant du vieux Sud américain, Natchez est passé dans l'oubli avec la guerre civile. Natchez-under-the-Hill, dans cette perspective, c'est l'envers de I'Ündensacre, ce lieu du spectacle où se joue le devenir du colonisé. Et toute âme colonisée qui, comme Sylvie, recule devant le mouvement libérateur. qui embrasse son amnésie culturelle pour se diriger vers le théâtre de l'oubli, découvrira, au terme du voyage, la scène de sa dissolution finale. C'est cette même dissolution que Sylvie a pressentie lors de sa crise dans la Ford Torino. "Tout s'est fracturé en moi, dira-t-elle à Nicolas. "Les parcelles de mon corps se décollaient l'une de l'autre... je sentais mon ventre se lézarder à l'intérieur... et sous mon crâne, je pouvais assister a ma propre dissolution" (pp. 72-73).

II n'est donc pas étonnant que toute recherche du mouvement libérateur, "cette symphonie du désir et de l'espérance," soit d'abord un travail de désaliénation, un affranchissement des cloisons nationales. La trajectoire symbolique en ce cas sera celle du voyage, échappatoire à la spatialisation de Montréal, "au-dessus du Nord du Québec" (p. 35), vers le mouvement du devenir. Si Nicolas décide d'aller en Norvège, pays de Fortınbras, c'est bien pour marquer "une coupure entre avant et après" (p. 16), entre la vie de comédien (la représentation) et celle de scénariste-réalisateur (la création). Cette insistance sur la relation avant-après n'est pas une simple valorisation sartrienne de l'ek-stase présente où la conscience qui se révèle à elle-mème dans un dépassement qui affirme son manque d'être se donne un point de départ. Car si, pour la conscience autonome. le présent est ce "creux du nonêtre indispensable à la forme synthétique totale de la temporalité," 14 s'il est conditionné par les deux autres dimensions temporelles en même temps qu'il les conditionne, le présent demeure cependant implicite pour la conscience colonisée. Ou encore, selon Hubert Aquin dans Neige noire: "Le temps est une vierge enceinte." Non pas qu'il y ait ici une valorisation de l'ek-stase future à la manière de Heidegger. Pour l'auteur, victime d'une tragique sous-historicité. l'ek-stase présente est l'ek-stase future et ce "fossé qui tient lieu de présent... ne peut être respecté qu'au prix du silence» (p. 123). C'est bien à partir de ce silence "historique" que la conscience du conquis devient son passé en tant qu'être "qui a à être son être sous la forme diasporique de la Temporalité. 15 C'est en ce sens que la Temporalité est la structure interne ("intra-structure") de la conscience subjective. En conséquence, si l'auteur invoque un voyage intérieur pour capter le temps, c'est parce que ce dernier "est le secret mème de la subjectivité» (p. 185). 
Toute recherche d'une unité qui se définit essentiellement comme à̀ la fois être et ne pas être» va donc nécessairement se jouer sur le théâtre tri-dimensionnel du temps. Trois dimensions, chacune ek-statique, où la conscience est pour ainsi dire séparée de l'être par le non-être, dans lesquelles Nicolas doit tour à tour séjourner pour finalement déboucher sur lunité de l'être. Mais accéder à l'unité pour Nicolas, c'est accéder à un mode d'existence qui se fonde sur le paradoxe d'avoir à exister simultanément dans le chassécroisé de toutes ces dimensions, c'est-à-dire à la fois dans le Présent, le Passé et le Futur, dans une perpétuelle fuite de l'être.

\section{"Le tempus continuatus de l'ancienne Thulè"}

La négation de l'être par le dépassement implique forcément que l'être qui est nié est un être d'avant, c'est-à-dire qui est l'identité originelle et qui appartient au passé. Nier l'être d'avant, dans cette perspective, entraine nécessairement un séjour dans le passé, ce négatıf dont l'homme tient son pouvoir et qui est "...puissance seulement en sachant regarder le négatıf en face, et en sachant séjourner près de lui. Ce séjour est le pouvoır magique qui convertit le négatif en ètre.»16 Aussi, quand Nicolas, en route pour le póle nord, fait escale à Trondheim, il découvre en feuilletant le journal anglass. le Times, que "ce dernier... le temps... ne peut être que rétrospectıf» (p. 58). Mais découvrir le temps rétrospectif. c'est découvrır le temps dans son élément essentiel et qualitatif : la durée, non plus le temps chronométré ou homogène. mais plutôt la durée hétérogène du mol, en dehors de toute perception quantitative ou spatiale. Si bıen que Nicolas, devant la vitrine d'un horloger à Trondheim, découvre que les pendules et les chronomètres sont arrètès (p. 59). C'est que, pour arriver au "tempus continuatus de l'ancienne Thulé" (p. 63), il faut comprendre que, "en dehors de mor, dans l'espace, il n'y a jamaıs qu'une position unique de l'aiguille et du pendule, car des positions passées il ne reste rien. 17

C'est bien cet èloignement de la mesure du temps qui permet à Nicolas d'entrevoır la puissance inexplorée du devenir québécois. Comme Sylvie. le Québec serait enlisé dans son passé, tous deux n'acceptant aucune présence. ni mème celle du souvenir. Chez Sylvie, en effet. l'impossibilité même de la présence est la présence. Et c'est cela justement qui définit le temps de "l'absence d'une présence" dont parle Aquin dans Neige noire, le temps où rien ne s'affirme et qui "ne représente plus rien." Mais, dira-t-ll, c'est un "seuil qui doit ètre franchi, sans quoi l'expérience ne peut être conclue" (p. 164). Et qu'est-ce donc que conclure l'expérience sinon dépasser ce plafonnement tonalı dont parle Aquin, ce phénomène d'entropie qui afflige la conscience du conquis? Comme le fait, lors du voyage à bord du Nordnorge, le choral glaciaire de la mer de Barents. symbole à peine déguisé du Québec, cette *symphonie dont la partition imprévisible rend toujours impossible son exécution... cet air informe qui contient tous les airs. ...qui continue 
de se faire entendre, mais ne raconte rien... cantate sans portée, sans registre» (p. 75), qui n'éclatera que sur le clavier de la lutte, lutte qui va jusqu'à la mort et qui est l'histoire. "Avec la mort commence la vie de l'esprit," dit Hegel. Ou encore, selon l'expression de l'auteur de Neige noire: «Vivre tue (p. 107). Et lorsque la mer de Barents se transforme en une violente tempète qui effraie Sylvie, cette même asymphonie océane change de registre: tout se déroule en majeur" (p. 76). C'est alors que Nicolas aperçoit par la lunette de pilotage le Sud du Spitzbergen où il semble n'y avoir aucune tempête. Quand la mer se calme. Nicolas et Sylvie regardent le Spitzbergen se rapprocher, illusion d'optique cependant, car c'est le Nordnorge qui s'approche de l'île. C'est dans ce cadre que le couple découvre l'unité.

L'on sait que l'Unité dialectique, étant avant tout un rapport du particulier à la totalité, est un devenir dans lequel le Sujet fini s'insère, c'est-à-dire un mouvement qui brise les cloisons. Mais Sylvie, comme l'écriture, "altère le mouvement de la vie» (p. 45). La tragédie de cette dernière, c'est son impuissance à franchir le seuil du "devoir être," ce Sollen qui la condamne à contempler un Absolu qui, comme le Spitzbergen, "s'élève au-dessus de la mer comme le spectre d'un continent refroidi : ... une jungle de glaciers et d'arêtes granitiques" (p. '82). II va de sol que dans ce cas l'Unité symbolique n'atteint aucune réalité et demeure symbolique. Effectivement, cette immersion partielle dans la mer de Barents qui communique "avec toutes les mers, avec tous les fleuves* figure un mouvement d'expansion qui s'arrête à Natchezunder-the-Hill (p. 81). C'est pourquoi le voyage du Nordnorge est "une exploration exaltée vers le pôle nord, non pas pour s'y rendre. mais pour s'arrèter juste avant, à proximité de la grande banquise qui flotte comme une barrière infranchissable autour de l'Absolu.... (p. 811. Le Nordnorge ne va pas plus loin dans le Billefjorden, il fait demi-tour et côtoie à nouveau des paysages devenus méconnaissables dans le sens inverse où "les ombres des cimes se sont déplacées comme sur un cadran solaire» (p. 89). Le voyage du Nordnorge n'est finalement qu'une ascension "en creux" de l'inaccessible, vers le sommet de ce "cône renversé" qui, selon l'auteur, encadre toute quête de l'Absolu pour le colonisé. Car, si Pyramiden n'a pas été aperç, si le Djebel Amour demeure “inconnu et inconnaissable" (p. 89), c'est que, comme Pyramiden, le Djebel Amour n'existe pas. Soi-disant dissimulé au sommet de la montagne appelée Djebel Druze, où les Druzes ont vaınement attendu pendant des siècles qu'il se révèle à eux, le dieu Fatimid - l'un des premiers chefs de la dynastie musulmane des Fatimides qui, avant sa mort, s'est proclamé Dieu réincarné - figure icı le faux dieu du conquis. Chose intéressante à noter, les Druzes de cette régıon ont cédé leur autonomie en 1944 pour finalement se fusionner aux Syriens sous un même gouvernement central. C'est dans ce sens que le sommet du "cône renversé" dont le Nordnorge n'a fait que parcourir la parabole, est, selon Aquin, le "vertex", non pas du "théâtre illuminé" que cherche Nicolas, maıs de ce mème "édifice fêlé" où se joue le devenir du colonisé. 
C'est donc sur les sommets du Spitzbergen, cette uîle irréellew \{p. 102\}. que Nicolas immole Sylvia au Djebel Amour. Du cimetière glaciaire, seul accès à lîle, jusqu'au refuge où le Spitzbergen se fige comme un gigantesque "reposoir de neige et de glace» (p. 106), c'est la vision suspensive du réel. "Tout est mat, plus rien ne réfléchit. Le paysage est aboli. La neige engendre une image de la réalité constituée autant par sa propre couleur que par l'absence mème de toute couleur ' (pp. 106-107). C'est le lieu où l'on pourrait dire que la neige est noire. Le Spitzbergen, dans ce sens, c'est l'Absolu sans le mouvement qui le réfléchit. c'est la pure Négativité qu'il faut détruire. dans I'intention du devenir. De sorte que, de toutes les morts possibles - "Sylvie a fait une chute dans une crevassen (p. 125), « Sylvie s'est suicidée sous mes yeux* (p. 128), ainsi que la noyade ratée dans le scénario - Nicolas opte pour le meurtre: a ...le meurtre vaut mieux que le suicide, car il dévoile une intention pure de destruction ( $(p$. 207). C'est un meurtre cependant qui demeure enfermé dans le blanc du texte. Ce n'est que dans le scénario que la véritable nature de la mort de Sylvie sera révélée. Or, le blanc du texte, c'est le silence et, a si le silence a une couleur, il serait noir» (p. 125). Comme les neiges éternelles du Spitzbergen qui dissimulent le corps de Sylvie, acouche plus épaisse ...d'année en année» (p. 129), le blanc textuel dans Neige noire est la négation ultime de l'être d'avant, ce "Soi du fini» qui doit disparaître afin d'arriver au Tout qui est vivant. Car, on s'en souvient, "Vivre tue."

\section{Point de fuite}

Avec Sylvie, meurt le passé enlisant que figurait son père, Michel Lewandowski. Et juste avant son suicide, ce dernier dira à Eva : "... je ne peux plus m'alimenter en souvenirs... l'amour a besoin de la mémoire pour s'approfondir et durer... Sylvie est morte ... elle $\mathrm{m}$ 'a entrainé dans le précipice avec elle... (p. 209). Le sacrifice de Syivie, dans cette perspective, c'est l'exorcisme de l'oubli, ce "trou de mémoire" qui afflige le Québécoıs, pour donner lieu au souvenir. "Sylvie n'était pas," dira Nicolas après la disparition de celle-ci, "elle a été. elle n'est plus, elle ne sera plus" (p. 131). Aussi, de retour à Trondheim, le souvenir d'un théâtre illuminé où se déroule le spectacle que cherche son autre double. Fortinbras, effleure Nicolas pour la première fois. C'est que le souvenir est pour Nicolas le seul accès à l'avenir: "demain relève de la mémoire» (p. 149). On se souvient que c'est grâce à la puissance du souvenir que le Prince de Norvège a revendiqué ses droits sur le Danemark: "I have some rights of memory in this kingdom, Which now to claim my vantage doth invite me.n (Hamlet, $V$, ii). C'est à cette même puissance du souvenir que Nicolas se réfère quand. mettant en questıon son propre être, il écrit: "Je n'étais pas, jai été, je me souviens, je ne suis plus..." (p. 131).

Or, dire "Je ne suis plus...." c'est admettre son manque d'être, c'est séjourner dans le négatif. C'est se présenter à Soi en tant que dissimulé ${ }^{18}$ ou masqué. Étape essentielle au devenir historique, le masque, dans Neige noire. est ainsi valorisé: 
.. les gens devraient aller au cinéma masqués.

(...) Le masque, visage de pure convenance, aurait pour effet de libérer le spectateur. (...)

L'idéal, même, serait d'imaginer le lecteur d'un livre portant un masque dont il pourrait même se servir comme signet. (p. 159)

Le masque, ici, c'est le lieu où commence l'activité de la conscience par laquelle le masque va devenir négation. Toute l'efficacité de Fortinbras se résume de cette manière. Admettant qu'il n'est plus ce qu'il a été, il révèle son identité et, ce faisant. il nie son masque ou son manque d'être. Dès lors. il est "la substance authentique, l'être ou l'immédiateté qui n'a pas la médiation hors de Soi, mais qui est cette médiation mème." 19 C'est dans ce sens que nier son manque d'être est la "négation de la négation."

Dire "Je ne suis plus...," c'est aussi séjourner dans ce «creux du non-être» qu'est le présent, séjour qui est une étape essentielle du devenir humain. Effectivement, nous le disions ci-dessus, cette négation de l'être qu'est l'ek-stase présente est avant tout un point de départ dont le masque serait le symbole le plus frappant. Cependant, ce présent demeure "en creux, " implicite pour le conquis. C'est pour aınsı dire le point de fuite qui aura lieu lors de la libération. «Membrane palatine entre ce qui vient de finir et ce qui n'a pas encore commencéx (p. 93), le présent du Québec, c'est ce présent qui, comme celui du scénario, n'est plus qu'un aprésent inexistentiel... un infinitif poreux» (p. 221). Aussi, le retour de Nicolas à Trondheim marque-t-il un retour au présent où “le temps a l'air d'un document antidaté " (p. 117). Et Nicolas, dans une tentative pour dater la mort de son épouse, se réfère encore une fois au journal anglais. le Times. Mais ales souvenirs imprimés ne coïncident pas avec les points de repère de Nicolas. J'étais demain, je serai hier. (...) Le temps, par cette opération caligariste, se réduit à la conscience du contre-temps" (p. 118). Dislocation du temps qui rappelle celle qu'éprouvait Hamlet: « The time is out of joint. (Ham/et, l, v.) et qui, dans le cas de Nicolas, indique un retour à la réalité présente et à celle, tragıque, du conquis. Le sacrifice de Sylvie, en exorcisant l'impuissance et l'inefficacité qui se rattache au tragique, n'a pas pour autant aboli l'angoisse de la double aliénation qui appartient a Nicolas. double en ce que ce dernier éprouve a la fois l'état ek-statique de l'être qui est celui de tout homme en voie de devenir et l'état de séparation d'avec le flux historique qui appartient a la conscience du colonisé. En fait, l'esprit hamlétien demeure une partie intégrante de la conscience de Nicolas. Ce dernier a mangé certaines parties du corps de sa victime et a sucé le sang des "muscles du chagrin," "liquide alcalin et légèrement salé qui ne le rassasie pas" (p. 244). Comme Fortinbras [ "For me, with sorrow, I embrace my fortune.» (Ham/et. $V, i i)$, c'est avec tristesse que Nicolas embrasse sa destinée. Aussi, de retour à son appartement de la rue Berri, à Montréal. Nicolas, appuyé à la fenêtre, écoute la représentation télévisuelle de la pièce de Shakespeare, "mais sans vraiment voir, à travers ses larmes, l'ouest de Montréal et le profil du Mont-Royal» (p. 168). Cette valorisation du tragique dans $N$ eige noire nous rapproche de la conception 
hégélienne pour qui l'Histoire est l'«autel où ont été sacrifiés le bonheur des peuples, la sagesse des Etats et la vertu des individus. $" 20$ Et si la petite valise de Nicolas devient le cercueil symbolique de Sylvie, c'est que I'Histoire est, selon l'expression hégélienne, la "vallée des ossements, " à partir de laquelle l'Absolu "s'élève de ses cendres à la majesté." 21 Ainsi, Nicolas tient le corps de Sylvie "encoffré près de lui, il le porte, mais le poids du corps l'entraîne, détermine désormais sa marche et sa destinée” (p. 120). C'est à partir de cette détermination que l'autre double de Nicolas, Fortinbras, entre en jeu.

La conscience dominante dès la mort de Sylvie est donc celle de Fortinbras. C'est le passage au mouvement qui est médiation. Jusqu'ici sans importance visible, émanant par le truquage de la caméra du regard de Nicolas (p. 87). Eva Vos est littéralement éveillée à Oslo par le coup de téléphone de ce dernier. Objectivation du Soi qui devient autre, la Norvégienne est le produit organique de la conscience déterminée et efficace. Eva est l'antıtragique. Là où devant le miroir, Sylvie a les yeux embués de larmes, Eva se sourit. A deux reprises, à la fin du roman, elle embrasse son image dans la glace, signifiant ainsi son refus du Destin tragique qui appartenalt à Sylvie. $\mathrm{Ce}$ ne sont pas les «muscles du chagrin" qui distinguent Eva: elle ne porte pas "le mal de vivre comme Sylvie. Eva a des muscles de mélancolie (traduction de l'auteur du norvégien du mot “chagrin»), mélancolie, on le verra, qui est nostalgie et qui mène au souvenir. C'est donc avec Eva que Nicolas sera en mesure de réaliser une intégration de sa propre négativité. Et quand, plus tard, dans un cauchemar où il joue Hamlet, le souvenir du "théâtre illuminé" se précise. Nicolas se trouvera dans une enclave italienne sur les bords de la mer de Barents, cette ville "construite selon un plan concentrique..." (p. 144). Pris de panique devant la circularité tragıque de la conscience hamlétienne, Nicolas sera incapable de terminer la représentation. C'est Eva qui lui dira: "Oublie cette histoire d'enclave italienne. II n'y a pas d'enclave italienne à l'intérieur du cercle polaire. .. (p. 144).

Or, ce même mouvement circulaire et tragique, dans la Phénoménologie, figurait le progrès historique, schéma qui, dans la dernière phase de la philosophie hégélienne (à partir de l'Encyclopédie) deviendra rectıligne. ${ }^{22}$ C'est justement ce mouvement rectiligne que Nicolas veut récuperer lorsqu if dit: "Natchez-under-the-Hill... Essayez de vous rappeler Eva" (p. 133). Se souvenir de Natchez-under-the-Hill, c'est sortir de la circularite entropique qui caractérise l'histoire de cette ville et en suivre l'unique rue qui longe le fleuve, car, «au bout de la rue, le marcheur peut entrer doucement dans les eaux du Mississippi et s'y baptiser par immersion jusqu'à ce que son corps purifié de l'esprit du mal se liquéfie et roule, comme les vagues du grand fleuve. jusqu'à Bâton Rouge. Plaquemine. Chalmette. Evergreen..." (p. 224). C'est en quelque sorte résoudre "l'équation tragique" de Syivie et marcher avec le "progrès rectiligne de la conscience de la liberté et de sa réalisation. ${ }^{23}$ C'est au cours de ce mème cauchemar, qui survient après l'union de Nicolas et d'Eva, que la conscience de Nicolas, grâce au souvenir. 
se séparera du mouvement entropique, de la circularité hamlétienne, pour l'intégrer, dans toute sa tragédie, a son Moi. Alors seulement il sera en mesure de dire : « Je me souviens, avec tristesse, du théâtre illuminé ... (p. 143).

Mais jusqu'ici, Nicolas est pour ainsi dire fixé dans sa puissance d'agir. Fixité du Moi x en opposition au contenu distinct," dirait Hegel, "soit la fixité des différences qui, posées dans l'élément de la pure pensée, participent alors à l'inconditionnalité du Moi. ${ }^{24}$ C'est qu'Eva n'est pas encore intégrée à la conscience de Nicolas. Elle représente le mouvement médiateur en extériorité au Moi («hors de Soi») dont parle Hegel. En conséquence de quoi. Nicolas et Eva reposent fraternellement. "immobiles, parallèles, près l'un de l'autre et ne se touchant pas... Eva tourne la tête vers lui, prenant soin de ne pas empiéter sur l'espace qui les sépare... (p. 136). C'est cette image des $\propto$ deux gisants, $"$ figures d'une même conscience différenciée, que l'auteur quitte pour écrire:

(Jusqu'à présent dans le film, on peut dire que le Québec est en creux. Son éclipse récurrente fait penser à l'absence d'une présence, à un mystère inachevé...) (p. 136).

Ce n'est que la nuit suivante que la détermination et l'efficacité d'Éva entrent véritablement en jeu et que l'union du couple a lieu. Nicolas et Éva sont tous deux «accoudés au balcon de la fenêtre. « Le balcon, point pivot en quelque sorte - on se souvient de l'importance de celui-ci dans Le Parc de Philippe Sollers et dans aLe Balcon * de Baudelaire - marque ici une transition. Effectivement, c'est le lieu où Nicolas assume finalement toute la tragédie de sa destinée:

\section{EVA}

La nuit dernière, je vous ai regardé vous endormır ... et vous avez parlé...

\section{NICOLAS}

Qu'est-ce que j'ai dit?

EVA

Je ne serai jamais plus capable d'aimer...

(pp. 136-137)

Aussi, la nature de l'échange sexuel entre Nicolas et Éva ne demeurera au cours de leur liaison qu'une participation "à un même délire d'obscuration et de plaisir" (p. 184).

On sait que, mouvement "partiellement déterminé," toujours à recommencer, Eva prolonge Nicolas. C'est donc elle qui se dirige la première vers la chambre où aura lieu leur liaison. Et lorsque la caméra se fige sur un gros plan de Nicolas, on entend Éva dire: "Je ne suis pas ta sœur, tu n'es pas mon frère...» $\langle p .137\rangle$. L'acte brutal qui suit n'est pas un acte de fusion amoureuse mais, écrit l'auteur: “les deux, entrainés par une précipitation inabrégeable, s'accrochent...» (p. 137). Cependant, en surimpression sur l'iris de Nicolas, la caméra capte l'image d'Éva en mouvement dans Lille Grensen, représen- 
tations visuelles qui, selon l'auteur, «figurent l'antimasquen (p. 139). Cet antimasque est la médiation hors de Soi * selon laquelle Nicolas récupère l'activité essentielle à l'intériorisation de cette même médiation. C'est là une étape du devenir qui confère à la conscience l'authenticité recherchée et partant, lui permet d'explorer l'avenir. Aussi, dans Neige noire. les représentations visuelles d'Éva s'opposent-elles au thème musical du film qui est introduit avec elles. C'est pourquoi la musique a naît du regard: elle le précède, mais ne l'accompagne pas» (p. 139). Elle est en quelque sorte le mouvement antérieur qui permet à la conscience d'intérioriser la médiation qui, autrement. demeurerait extérieure ("hors de Soi»). C'est dans ce sens que "Eva, I'antimasque, ne fait que prolonger dans Lille Grensen I'hymne orphique qui lui est dédié... Le film, pendant ce passage... s'intériorise. Quand cesse le masque d'Éva, la suite du film reprend (p. 139). Et lorsque recommencent les caresses d'Éva, c'est «comme si la tempète qui vient de s'abattre ne faisait que se lever au large * (p. 138). Une exploration de l'avenir est ainsi amorcée. C'est là une étape essentielle du devenir historique, car, nous dit l'auteur, "l'esprit qui explore l'avenir est l'àme intérieure de tous les individus» (p. 194).

\section{Le «point oméga"}

L'écriture, à partir de l'union du couple, est valorisée. Structurellement, le scénario de Nicolas, inséré et subordonné au commentaire discursif qui en est la «châsse," devient comme "the play within the play" mais a l'envers: "non pas une insertion du plus petit dans le plus grand mais du plus grand dans le plus petit" (p. 195). De sorte que l'on retrouve ici un phénomène de resserrement caractéristique de l'écriture, "tandis que la réalisatıon cinématographique implique une dilatation. À choisir entre ces deux mouvements, on a tendance à n'élire le resserré que dans la mesure où Il est dilatable, écartant d'emblée ce sur quoi ne peut s'exercer aucune action" (p. 195). Le scénarıo n'est cependant pas encore réalisé et c'est seulement comme film qu'il sera pleinement imbriqué dans le commentaire discursif, c'est-à-dire que "le récıt ne sera totalement enchâssé que lorsque la châsse sera devenue invisible» (p. 196). C'est donc dans l'action exercée sur le scénario que l'élément discursif va disparaitre, élément qui, en ce sens, est le masque d'un scénario qui a "à être" dans un film à venir, tout comme ce même élément est le masque du roman qui a "à être" dans l'action future du lecteur. ${ }^{25}$ Et si l'action exercée par le cinéaste sur la «texture commentative» va métamorphoser le scénarıo en film, effectivement, l'activité du lecteur sur cette mème texture va métamorphoser le roman-scénario en une œuvre qui ira s'insérer, comme le film. dans «une étude ininterrompue sur Undensacre» (p. 196)

C'est pourquoi Nicolas éprouve ce devenır infini dans et par le scénario: «Je n'écris le scénario qu'à mesure que je le vis... et je n'al pas fini de le vivre dans la mesure où il est inachevé (p. 148). Et, dans la scène sans doute la plus puissante de Neige noire, où l'Hamlet de Shakespeare est représenté à la télévision, l'on retrouve ce même inachèvement du devenir humain. La 
représentation prend fin, alors que les caresses des deux amants qui regardent le petit écran n'aboutissent pas. De cette manière, a au lieu de se répandre dans la tunique muqueuse d'Éva, le nectaire (sic) s'écoulera dans les voies serpigineuses de l'imagination du spectateur et remontera jusqu'aux chapiteaux de l'être... (p. 177). Le « nectaire » s'écoulera hors de l'emprise du temps, ce temps qui n'est plus qu'un «ventre consentant» et qui emprisonne un spectacle aqui ne peut être donné puisque tout se passe. dans la noirceur humide du ventre...* (p. 172). C'est au cours de cette même scène que Nicolas dit: $\propto$ J'ai le sentiment d'aller jusqu'au bout de ton ventre.... Comme en écho, a la télévision, Nicolas, dans le rôle de Fortinbras, demande : «Où donc est ce spectacle?» (p. 175). Pour que le spectacle ait lieu, par conséquent, l'être doit demeurer perpétuellement dans un état d'inachèvement, sur la voie d'un devenir infini qui se joue dans «l'inévitable spatialisation du temps " que mème l'écriture ne peut transcender. Car si l'on arrivait à transcender le temps, on risquerait de "sombrer dans une crevasse spatiale» (p. 186).

Par surcroît, l'irréductibilité de l'espace qui sépare l'être de la personné aimée le situe forcément en dehors du temps intérieur de l'autre : "Personne ne connait personne, décidément... (p. 187). C'est là certainement le cœur du tragique humain, ce «mal d'être sur l'écorce impénétrable du réel* (p. 187). En proie a la lucidité de l'homme qui confronte son paradoxe insoluble. Nicolas écrit: "La cantate en miroir vient de se fracasser; ne subsistent, sous l'action dévastatrice du cogito cogitatem (sic), que les tessons d'un miroir sans tain !... il ne reste plus qu'a inventer un substitut musical pour exprimer cette grande désolation qu'on ressent: une cantate pour la vallée de la mort" (p. 187). Et sous la marche du temps pour ainsi dire à reculons. Sylvie, "revenante, " inverse la chorégraphie de sa chute dans le précipice, annulant l'image de cette chute qui, en ce cas, n'est plus "qu'une absence, le négatif d'un fantôme qu"on chérit " (p. 197). Cependant, toute l'action de Nicolas s'exerce précisément sur le négatif de l'être. A l'exemple de Fortinbras, c'est la mémoire de ses adroits sur ce royaume* (p. 193) qui confère à Nicolas la puissance nécessaire pour franchir ce «seuil ainsi que l'efficacité par laquelle la détermination de Nicolas devient finalement action revendicatrice.

C'est alors que le réel et l'imaginaire, jusqu'ici à l'envers, coïncident à la fois dans l'espace et dans le temps pour s'affranchir entièrement, à la fin de Neige noire, de ce schéma qui, selon Patricia Smart, serait caractéristique de la fatigue culturelle dont est affligé le conquis. ${ }^{26}$ A la recherche d'un lieu pour une scène qui n'est pas encore inscrite dans le scénario. Nicolas et Éva découvrent la maison dans lîle des Sceurs, où il y a une gravure de Natchezunder-the-Hill. C'est la maison réelle de Michel Lewandowski où l'inceste a eu lieu. C'est aussi la maison de ce dernier où une scène d'amour entre Sylvie et son père, inscrite dans le présent du scénario, se déroulera dans le film á venir. Grâce à cette fausse simultanéité (ou truquage temporel). la gravure de Natchez-under-the-Hill, dans un plan inversé, se transforme sous le regard de Nicolas, tout comme l'image de la chute de Sylvie dans le précipice, en ason 
propre négatif." Lorsqu'Éva découvre dans le scénario la véritable nature de la mort de Sylvie et en avertit Linda Noble, choisie par Nicolas pour jouer le rôle de Sylvie dans son film, le schéma du réel et de l'imaginaire est finalement inversé. L'écriture débouche alors sur une réalité qui «est contaminée par la fiction" (p. 220).

Que ce soit le négatif de la chute de Sylvie dans le précipice ou le négatif de la gravure de Natchez-under-the-Hill, c'est bien sur celui-ci que s'exerce l'activité du'devenir. En effet, Linda, grâce à la médiation d'Éva, refuse l'offre de Nicolas, refusant ainsi le Destin tragique de Sylvie, car le film, on le sait, est irréalisable sans un nouveau meurtre. Justement, c'est dans le rôle d'Ophélie dans la représentation télévisuelle de Ham/et qu'Éva aperçoit Linda pour la première fois. Masquée en quelque sorte. Linda a les yeux de Sylvie: "le bleu en est tellement pâle que tout l'ceil paraît blanc" (p. 169). Plus tard cependant, lors de leur véritable rencontre, Éva découvre que Linda a «les yeux sombres* (p. 230). C'est donc par ce mème processus de travail sur la négation que Linda récupère son effectivité et sa vérité, ce qui dès lors lui permet de se présenter comme "propriété de la conscience." 27 En quittant l'instabilité du Destin tragique, Linda s'achemine finalement, avec Éva, vers une unité synthétique “dans un présent qui se prolonge jusque dans son propre néant toujours renouvelé» (p. 251).

II est certes évident que la scène lesbienne qui termine Neige noire dépasse «le seuil de l'invraisemblance" (p. 253), et que c'est uniquement par une conciliation aqui récupère les oppositıons en les niant»que le lecteur pourra se diriger vers le "point oméga dont parle l'auteur. Ce terme. emprunté a Teilhard de Chardin, signifie a la fois le Christ et le terme final de la révolution. Révolution pourtant encore à faire car, si Nicolas "part demain pour Repulse Bay» (p. 247) afin de réaliser son film, le roman, lui, se termıne avant "demain. " La seule action possible, en ce cas, c'est celle qui va réaliser la (re)naissance historique d'un peuple conquis, peuple qui, seulement alors, sera en mesure de vivre pleinement dans l'Histoıre.

Le Québec colonisé, comme Sylvie, comme Natchez-under-the-Hill, est donc, aux yeux de l'auteur, un lieu d'impuissance et d'inefficacité. C'est ce théâtre qui "n'est plus illuminé," n'est même plus un théâtre: "L'édifice est fêlé et comme réduit à n'être que sa propre ruine... (p. 227). Mais c'est précisément ce "collage tragique» qu'il faut nier pour arriver au "point oméga que l'on n'atteint qu'en mourant et en perdant toute identité pour renaitre et revivre..." (p. 254), et qui, pour le Québec, est aussi le "point de fuite où l'action puise son départ. Repulse Bay, territoire canadien anglais, cet "enfer de blancheur et de silence» (p. 174), où Nicolas va réaliser la scène sacrificielle de son film, c'est le Spitzbergen de la conscience québécoise où la tragédie nationale sera pour ainsi dire expulsée. C'est le lieu d'une promesse à réaliser, où commence, pour le conquis, la véritable récapitulation "des étapes essentielles du devenir historique. ${ }^{28}$ 
Nul doute que la question du nationalisme québécois est, comme Sylvie au début du roman, la astructure porteusex de Neige noire. De là "stase languissante " qui paralyse Montréal dès lé début du roman, au «baiser final qui est infini o des dernières pages, c'est une conscience colonisée qui indique au lecteur québécois l'unique voie à suivre, celle de l'Undensacre. Tout le roman est une tentative pour échapper à la "parabole» d'un cône renversé, réalité «en creux» du Québec actuel, afin de rejoindre le devenir historique. C'est ce devenir qui embrasse Nicolas, Éva et Linda dans une seule et mème conscience et qui, une fois l'action révolutionnaire accomplie, ira s'inscrire dans l'Histoire, cette autre aparabole» qui se joue dans le temps, sur le théâtre illuminé où «toutes les œuvres humaines sont enchâssées» (p. 154).

1. Hubert Aquin, Neige noire, Montréal, Les Editions La Presse, 1974, p. 41 . Nous n'indiquerons désormais que la référence paginale.

2. Hegel, Wissenschaft der Logik, 1812, 1816. Ed. Lasson, F. Meiner, 1946, Trad. S. Jankélevitch: La science de la logique, éd. Aubier, pp. 37-38.

3. Hegel, La Science de la logique, pp. 37-38.

4. Hegel, Philosophie des Rechts, 1821, Jubiläumsausgabe, vol. VII. Trad. A Kaan. éd. Gallimard, 1940, p. 31.

5. Cet *ètre-autre» n'étant effectivement jamais extérıorisé, il va de soi que la conscience qui s'objectifie de cette manière va en garder les mêmes traits. La ressemblance étonnante entre les personnages de Neige noire peut certes s'expliquer ainsı. Par exemple, lorsque Nicolas imagine Sylvie dans les bras d'un partenaire qui se révélera être nul autre que son père. Michel Lewandowskı, Nicolas dit: "Malheur à moi d'avoir vu, de voir. ce que j'ai vu. ce que je vols!’ (p. 22). Plus tard, ces mêmes paroles seront répétées par Linda à la télévision dans le rôle d'Ophélie (p. 169). Quant à Sylvie, qui représente toute la négativité de la conscience hamlétienne, les similitudes entre elle et les autres personnages abondent dans le texte.

6. Hegel, Phanamenologie des Geistes, 1807. Ed. F. Meiner, 1949. Trad. Jean Hyppolite: La Phénoménologie de l'Esprit. éd. Aubier, p. 26.

7. Kierkegaard, L'Existence, Paris, Presses universitaures de France, 1972, p. 87.

8. Kierkegaard, L'existence, p. 117.

9. Hubert Aquin, Trou de mémoire. Montréal, Le Cercle du Livre de France, 1968, p. 56.

10. Marcel Régnier, uHegelo dans Histoire de la Philosophie 2. Encyclopédie de la Pléiade, Paris, Gallimard, 1973, p. 860.

11. Henri Bergson, Essat sur les données immédiates de la conscience, Paris, Presses universitaires de France, 1927, 1970, p. 81.

12. Bergson, Essai... p. 75.

13. Hubert Aquın, Prochain épisode, Montréal. Le Cercle du livre de France. 1965. p. 10

14. Jean-Paul Sartre, L'être et le néant, essai d'ontologie phénoménologique, Paris, Gallimard, 1943, 1960, p. 188.

15. Jean-Paul Sartre, L'étre et le néant..., p. 188.

16. Hegel, Phénoménologie.... p. 29.

17. Bergson, op. cit., p. 80.

18. Voir à ce sujet "La solitude essentielle et la solitude dans le monde, dans L'espace littéraire de Maurice Blanchot, Paris, Gallimard, 1955, pp. 341-344. 
19. Hegel, La Phénoménologie..., p. 29.

20. Hegel, Die Bernunft in der Geschichte, éd. Hoffmeister, F. Meiner, 1955, p. 80.

21. Hegel, Theologische Jugendschriften, éd. Nohil, 1907, p. 380.

22. Kostas Papaioannou, Hegel, Paris, Editions Seghers, 1962, p. 75.

23. Kostas Papaioannou. Hegel, pp. 74-75.

24. Hegel, La Phénoménologie.... pp. 30-31.

25. Ce point de vue diffère de celui de Françoise Maccabée-labal dans son étude intitulée "L'appel du Nord dans Neige noire: La quête de Narcisse " (Voix et images. vol. V, $n^{\circ}$ 2, hiver 1980 , pp. 365-377), où elle cite (p. 366) une remarque d'Eva "The play within the play, comme dans Hamlet... Mais a cela. Nicolas répond: "Non... Hamlet a inventé le stratagème de la pièce pour piéger Claudius. Dans mon scénario, la fiction n'est pas un piège. c'est elle. plutòt qui est piégée par une réalité qu'elle ne contenait pas et qui l'envahit hypocritement... Nerge noire. p. 147).

26. Patricia Smart, Hubert Aquin. agent double, Montréal, Presses de I'Université de Montréal, 1973, p. 111.

27. Voir Hegel, Phénoménologie.... p. 33.

28. Marcel Régnier, "Hegel, Historre de la Phılosophie..., p. 857. 